\title{
DISTRIBUTION AND NATURAL HISTORY OF THE WORKERLESS INQUILINE ANT POGONOMYRMEX ANERGISMUS COLE (HYMENOPTERA: FORMICIDAE)
}

\author{
By ROBERT A. JOHNSON \\ Department of Botany \\ Arizona State University \\ Tempe, Arizona 85287-1601
}

\begin{abstract}
Seven colonies of the rare inquiline Pogonomyrmex anergismus Cole were located in New Mexico, two in Hidalgo County, and five in Grant County; P. rugosus was the host for each. Mating flights follow late summer rains. Males mated with colony-mates, mostly inside the nest, and then returned to the nest. Pogonomyrmex anergismus may parasitize host nests over multiple years, with presence of host female alates confirming that the host gyne is not killed. Observations suggest this may be a polygynous species.
\end{abstract}

\section{INTRODUCTION}

Pogonomyrmex anergismus Cole is one of two inquiline species known in its genus (Snelling 1981, Rissing 1983). This species is known from three locales, the type locality 15 miles east of Silver City, New Mexico (Cole 1954), the Chiricahua Mountains of southeastern Arizona (MacKay and Van Vactor 1985), and Buffalo Lake State Park, Lubbock County, Texas (specimen in Los Angeles County Museum of Natural History, host unspecified). Hosts at the first two locales were $P$. rugosus and $P$. barbatus, respectively. This paper presents additional location records and observations on natural history of $P$. anergismus.

\section{OBSERVATIONS}

Five colonies of $P$. rugosus parasitized by $P$. anergismus were located 300-1000 m south of Interstate 10 between 6.1 and $7.6 \mathrm{~km}$ east of Separ, Grant County, New Mexico (32 $10^{\prime} 30^{\prime \prime N}$,

Manuscript received 3 March 1995. 
$108^{\circ} 21^{\prime} 30^{\prime \prime} \mathrm{W}$ ), on November $1-2,1993$. Two parasitized colonies were located $0.5 \mathrm{~km}$ west of the junction of Highways 9 and 80 , Hidalgo County, New Mexico (31 $55^{\circ} 30^{\prime \prime N}, 109^{\circ} 2^{\prime} 30^{\prime \prime W}$ ), on July 27, 1994 and July 17, 1995. The site east of Separ was a Hilaria mutica grassland, with washes in the area also containing Yucca elata and Ephedra sp.; elevation was $1375 \mathrm{~m}$. Vegetation at the Hidalgo County site was dominated by Flourensia cernua; elevation was $1265 \mathrm{~m}$.

The Separ colonies were found by making shallow excavations into nests of $P$. rugosus and $P$. barbatus (similar to the original discovery of the species by Cole [1954]). The colonies in Hidalgo County were located while digging for $P$. rugosus alates, which typically inhabit the uppermost nest chambers during early morning; individuals of $P$. anergismus were found in these chambers intermixed with host alates. Pogonomyrmex anergismus occurred at very low densities, as these were the only parasitized colonies out of several hundred host colonies examined at each site.

Mating flights of $P$. colei Snelling, the other Pogonomyrmex inquiline, and most species in the genus follow summer rains (Hölldobler 1976, Rissing 1983, Johnson et al., in review), suggesting that $P$. anergismus may respond to the same environmental trigger. Attempts to induce mating flights were made by pouring about eight liters of water on parasitized colonies, a procedure which often induces flights in other Pogonomyrmex. The host colony in Hidalgo County was watered on July 28 and 29, 1994; a light rain also occurred on the night of July 28 . I briefly saw one $P$. anergismus individual at $1415 \mathrm{~h}$ on July 29; both host alate sexes were observed at this time, and host alates flew later that afternoon. A heavy rain occurred on August 9 and the same colony was observed intermittently the next afternoon; host alates again flew from the colony, but no individuals of $P$. anergismus emerged from the entrance. Water was also poured on three parasitized colonies near Separ at $0900 \mathrm{~h}$ on September 20. A P. anergismus mating flight occurred at one colony from the start of observations at 1200 $\mathrm{h}$, until about 1430-1445 h; one $P$. anergismus female also emerged from a second colony during this period. During mating activities, workers of $P$. rugosus aggressively defended the nest area such as occurs during mating flights of conspecifics. 
Like many inquiline species, males of $P$. anergismus mate with colony-mates. Few males were observed outside the nest, and females often left the nest and flew without contacting males, which suggest that most mating occurs inside the nest. Spermathecae of three such females captured flying from the nest appeared full (B. Hurley, pers. comm.), and contained an average of 8,200 sperm (range 8,000-8,500). As in several free-living congeners (Hölldobler 1976), P. anergismus females are capable of mating with multiple males. Such behavior was determined by placing several females that were leaving the nest in a small plastic container with several males; most females were in copulo within minutes. Males did not fly, but remained on the ground near the entrance and later returned to the nest. A dealate $P$. anergismus female, perhaps the nest gyne, was also observed periodically outside the nest during the flight.

Attempts to introduce mated $P$. anergismus into laboratory colonies of $P$. rugosus were mostly unsuccessful due to high levels of worker aggression. Host workers were also immediately hostile, e.g., stinging and grabbing legs with mandibles, during the one successful introduction. However, when the gyne made contact with the host gyne and/or her brood, hostile behavior was replaced by intensive grooming by host workers. Thereafter, the $P$. anergismus gyne remained proximate to or on top of and frequently groomed the P. rugosus gyne (C. Rodriquez, pers. comm.). Both gynes survived for about three months, with no apparent hostility after acceptance; the $P$. anergismus gyne then died, still after an apparent lack of hostility.

Pogonomyrmex anergismus may parasitize host colonies for multiple years, as at least two of the five colonies located in 1993 were still alive in 1994. Accordingly, P. anergismus gynes do not kill the host gyne, as host female alates were captured from three colonies (one in the second year of observation). Two dealate $P$. anergismus females were observed in two host nests. Throughout this study, no individuals were observed that could have been $P$. anergismus workers.

Voucher specimens are deposited at the Museum of Comparative Zoology at Harvard University, Los Angeles County Museum of Natural History, and the collections of R. A. Johnson and W. P. MacKay. 


\section{Discussion}

Pogonomyrmex anergismus appears to be very rare, as only seven of more than several hundred host colonies were parasitized at each of two sites. This is the first record of host female alates in parasitized nests; their presence in the second year of observations confirms that host gynes survive invasion by $P$. anergismus. Consequently, $P$. anergismus may parasitize host colonies for multiple years. Lack of host alates in the type colony during mid-September (Cole 1954), most likely after completing their mating flight, resulted in Nonacs and Tobin (1992) assuming incorrectly that host females do not survive parasitism by $P$. anergismus.

Mating flights of $P$. anergismus are triggered by rain. Lack of response to artificial watering in July and August suggests that mating flights typically occur later than those of the host, apparently in September and October. This is supported by locating the Chiricahua Mountains colony on October 7 and the Lubbock, Texas, specimen on October 19; the latter collection was of a single female, most likely captured after flying from the natal colony. Consequently, emergence of host and inquiline sexuals may be offset, as those of both hosts are present by mid-June, while the Hidalgo County colony contained $P$. anergismus males that had just emerged from the pupal stage in late July. Occurrence of mating flights after those of both host species makes it likely that only host colonies are invaded, and that $P$. anergismus gynes do not attempt to parasitize foundresses. Host colonies may be located by following trail recruitment phermones, as is the case for $P$. cole $i$ (Johnson et al., in review).

Mating with nestmates is common in rare ant species such as $P$. anergismus, and presumably evolved to insure mating when probability of outcrossing is extremely low (Wilson 1963). Occurrence of mating within the nest would represent the most highly derived mating system in the genus, even compared to $P$. colei, in which males congregate outside the nest and mate with females as they emerge from the entrance (Rissing 1983, Johnson et al., in review). That the mating system of $P$. anergismus may be the most highly derived in the genus is also indicated by exceedingly low sperm counts in mated females, which average 20 -fold lower than those of $P$. colei (R. Johnson, unpub. data). Discovery of two dealate females in two colonies suggests that colonies may often have 
multiple gynes. Based on the observed extremely low nest parasitism rates, occurrence of multiple females may be caused in part by mated females re-entering the natal colony.

As in $P$. colei, host workers are the primary barrier preventing acceptance of inquilines into host colonies (Johnson et al., in review). Rapid change of worker behavior after the P. anergismus gyne contacted the host gyne and/or her brood suggests that she acquired the colony odor, perhaps by licking the host gyne or brood.

Observing a dealate $P$. anergismus female outside the nest during mating activities seems highly unusual. Such risky behavior might be related to extremely low sperm counts, which are three orders of magnitude lower than that of host gynes (R. Johnson, unpub. data). Consequently, one explanation for this behavior, though such was not observed, is that gynes participate in mating activities and replenish sperm supplies by remating with sons. Alternatively, very low sperm counts and risky behaviors, along with an observed three month longevity in a laboratory colony, may indicate that $P$. anergismus gynes are short-lived.

\section{ACKNOWLEDGEMENTS}

I thank S. P. Cover, the Social Insect Research Group at ASU, and two anonymous reviewers for comments that improved this manuscript.

\section{Literature Cited}

Cole, A. C. 1954. Studies of New Mexico ants. VII. The genus Pogonomyrmex with synonomy and a description of a new species (Hymenoptera: Formicidae). J. Tenn. Acad. Sci. 29:115-121.

Hölldobler, B. 1976. The behavioral ecology of mating in harvester ants (Hymenoptera: Formicidae: Pogonomyrmex). Behav. Ecol. Sociobiol. $1: 405-423$.

Johnson, R. A., J. D. Parker, and S. W. Rissing. Distribution and additional natural history of the workerless inquiline ant Pogonomyrmex colei (Hymenoptera: Formicidae). Ins. Soc., in review.

MacKay, W. P., and S. Van Vactor. 1985. New host record for the social parasite Pogonomyrmex anergismus (Hymenoptera: Formicidae). Proc. Entomol. Soc. Wash. 87:863.

Nonacs, P., and J. E. Tobin. 1992. Selfish larvae: development and the evolution of parasitic behavior in the Hymenoptera. Evolution 46:1605-1620.

Rissing, S. W. 1983. Natural history of the workerless inquiline ant Pogonomyrmex colei (Hymenoptera: Formicidae). Psyche 90:321-332. 
Snelling, R. R. 1981. The taxonomy and distribution of some North American Pogonomyrmex and descriptions of two new species (Hymenoptera: Formicidae). Bull. S. Cal. Acad. Sci. 80:97-112.

Wilson, E. O. 1963, Social modifications related to rareness in ant species. Evolution 17:249-253. 

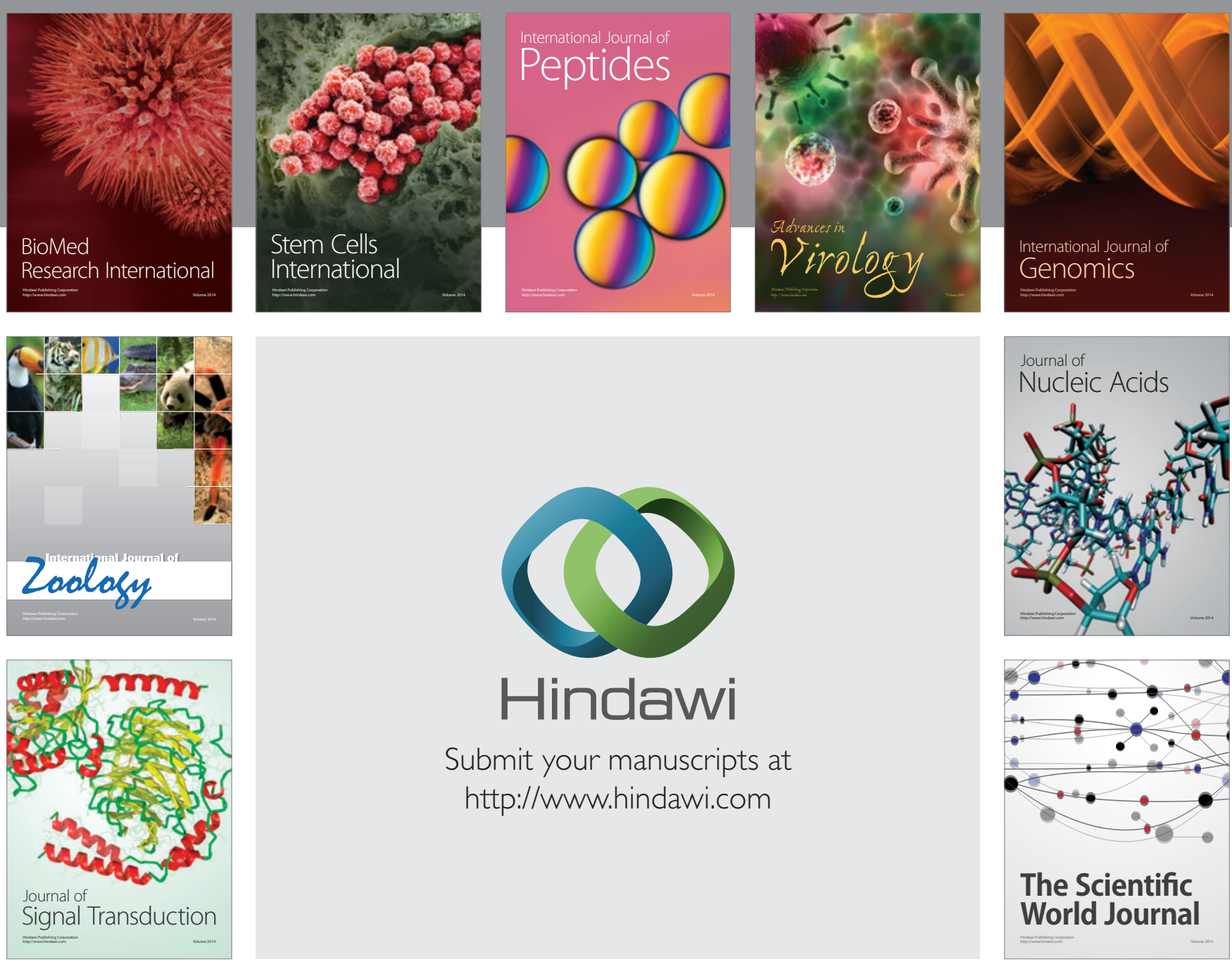

Submit your manuscripts at

http://www.hindawi.com
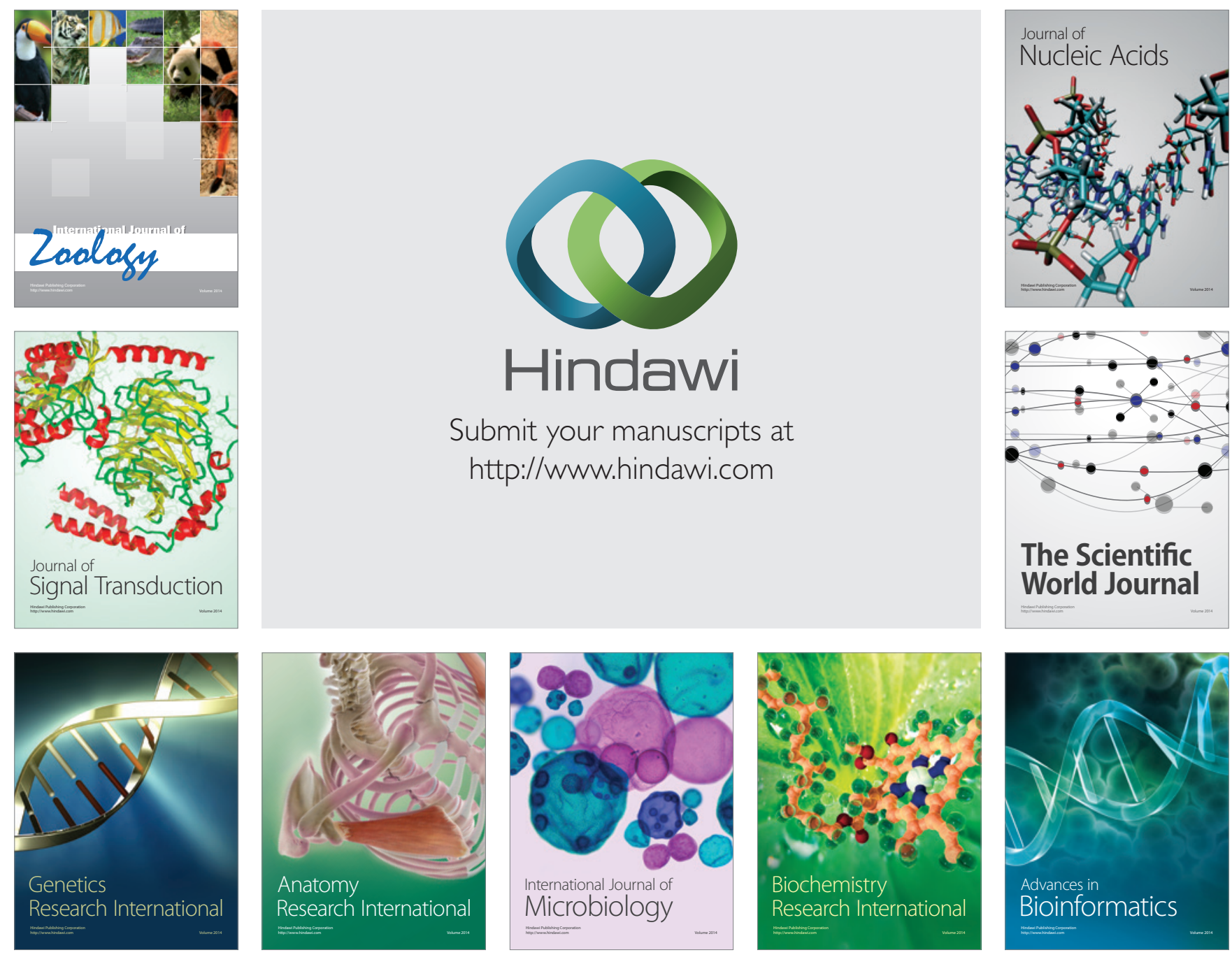

The Scientific World Journal
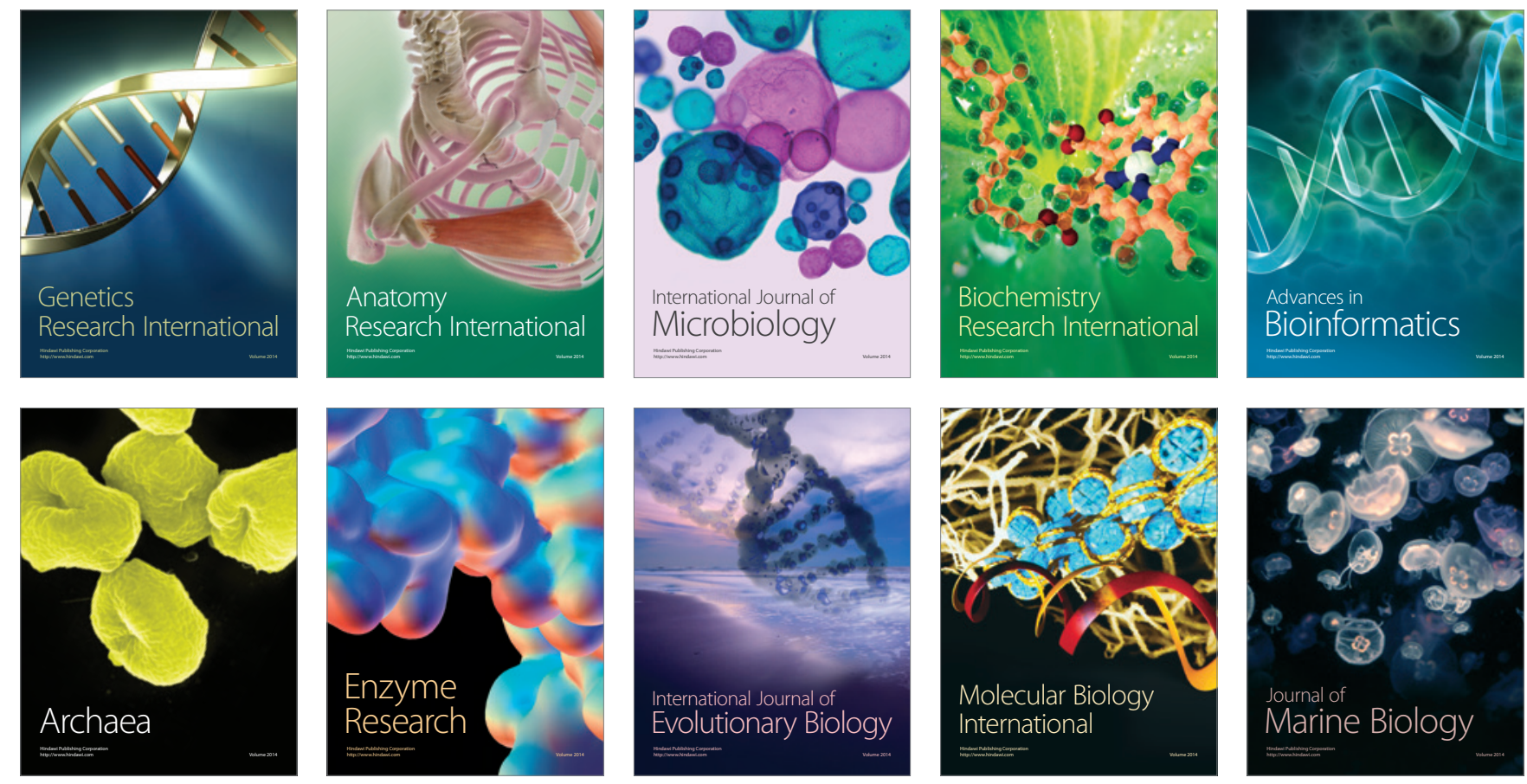